\title{
In Situ Control of Gas Flow by Modification of Gas-Solid Interactions
}

\author{
Dongjin Seo and William A. Ducker* \\ Department of Chemical Engineering, Virginia Tech, Blacksburg, Virginia 24061, USA
}

(Received 24 June 2013; published 25 October 2013)

\begin{abstract}
The boundary condition for gas flow at the solid-gas interface can be altered by in situ control of the state of a thin film adsorbed to the solid. A monolayer of ocatadecyltrichlorosilane (OTS) reversibly undergoes a meltinglike transition. When the temperature of an OTS-coated particle and plate is moved through the range of OTS "melting" temperatures, there is a change in the lubrication force between the particle and plate in $1 \mathrm{~atm}$ of nitrogen gas. This change is interpreted in terms of a change in the flow of gas mediated by the slip length and tangential momentum accommodation coefficient (TMAC). There is a minimum in slip length $(290 \mathrm{~nm})$ at $18^{\circ} \mathrm{C}$, which corresponds to a maximum in TMAC (0.44). The slip length increases to $590 \mathrm{~nm}$ at $40{ }^{\circ} \mathrm{C}$ which corresponds to a TMAC of 0.25 . We attribute the decrease in TMAC with increasing temperature to a decrease in roughness of the monolayer on melting, which allows a higher fraction of specular gas reflections, thereby conserving tangential gas momentum. The importance of this work is that it demonstrates the ability to control gas flow simply by altering the interface for fixed geometry and gas properties.
\end{abstract}

PACS numbers: 47.85.mf, 34.35.+a, 47.45.-n, 47.45.Gx

Gas flows are usually calculated from the known geometry of the container, the properties of the gas, and the known or assumed boundary conditions. If the boundary condition becomes a variable that could be user controlled, then it is possible to alter gas flow in applications such as flow control in small radius tubes or perhaps flight control for very small objects, for a constant geometry and gas conditions. In this Letter we demonstrate in situ reversible control of gas flow in a narrow channel by changing the state of a film adsorbed to a solid surface. Our demonstration uses a chemically adsorbed monolayer of octadecyltrichlorosilane (OTS) on smooth glass. The hydrocarbon octadecane melts at about $28^{\circ} \mathrm{C}$ [1]. OTS is similar to octadecane but can be covalently tethered to silica. When tethered, it undergoes a transition akin to melting whereby there is an increase in the ratio of gauche:trans conformations over a temperature range of $0-100^{\circ} \mathrm{C}$ [2-4]. By "melting" an OTS film, we aim to change the conservation of gas tangential momentum when gas molecules collide with the surface, and therefore to alter the flow of gas. The importance of the conformational change is that the high temperature "liquid" form is expected to be smoother, thereby promoting specular reflection. The high temperature form may also be less stiff, which would decrease the fraction of momentum conserved by the gas.

Temperature-induced melting is a rather clumsy method of control, but we believe that this is the first demonstration of stimulus-response surface controlled flow, and shows the route for more elegant systems such as electrical fieldor light-activated flow control.

For macroscopic systems, it is usually accurate to assume the no-slip boundary condition at the gas-solid interface. This assumption has diminishing accuracy at high Knudsen number, $\mathrm{Kn}=\lambda / l$, where $\lambda$ is mean free path, and $l$ is characteristic length of the system, i.e., at low pressure or for thin gas films. Applications at atmospheric pressure include micro-electro-mechanical systems and nano-electro-mechanical systems [5], as well as lab-on-achip systems. Kn in the range of $0.1-0.001$ is usually described as the slip-flow regime [6-8], where partial slip occurs. The magnitude of partial slip $b$ was described by Maxwell in terms of a tangential momentum accommodation coefficient (TMAC), $\sigma$ [9]:

$$
b=\lambda\left(\frac{2}{\sigma}-\sigma\right) .
$$

If a surface accommodates all the tangential momentum from a colliding gas molecule then $\sigma=1$, giving the minimum slip length of $b=\lambda$. If all the gas tangential momentum is conserved, then $\sigma=0$, and $b=\infty$. Since Maxwell, many refinements of Eq. (1) have been suggested $[5,10]$; it is important to note that in this Letter, these refinements will affect our measured values of $\sigma$, but not our conclusions [11].

Recent work has shown that gas flows can be measured using atomic force microscopy (AFM) [12] colloid probe measurements [13,14]. Laurent et al. [15] used lubrication measurements to show a dramatic increase in fitted slip length at low pressure, as expected from Eq. (1). Using similar AFM measurements, Lissandrello et al. [16] examined damping over a range of low pressures, and developed a scaling function to describe damping in terms of mean free path, radius, separation, and the viscous boundary layer thickness. Honig et al. [17] showed that adding a molecularly thin film of silane to glass caused a decrease in $\sigma$. Seo et al. [18] showed that the formation of water films on a glass solid alters $\sigma$ at $1 \mathrm{~atm}$ of $\mathrm{N}_{2}$. For example, the TMAC on equilibrium water films at $10 \%$ relative 
humidity $(\mathrm{RH})$ are greater than at $0 \%$ humidity, and the TMAC is lower at $88 \% \mathrm{RH}$. These two demonstrations of altering the TMAC by addition of a film suggest the possibility of altering the TMAC in situ by manipulating the properties of a film. This is the objective of the current work. Accommodation coefficients for various gases have also been determined by measuring the gas flow and pressure difference along microchannels. These measurements often find accommodation coefficients of about 0.85 on silicalike materials $[19,20]$ which is greater than the values reported in this Letter. The difference is that the solids considered here are much smoother and the humidity is kept at $0 \% \mathrm{RH}$.

The flow of gas in thin films was determined by the indirect method of measuring the lubrication force between a glass sphere (radius, $r \sim 15 \mu \mathrm{m}$ ) and a flat glass plate, using the AFM colloid probe technique [18]. In short, both the sphere and the plate were cleaned in oxygen plasma, coated with OTS monolayer in hexadecane solution, and then sonicated in toluene to remove any impurities. The sphere and plate were placed in a sealed chamber; the chamber was purged with dry $\mathrm{N}_{2}$ at $1 \mathrm{~atm}$. Because previous work showed that the lubrication force was a function of humidity [18], we kept the humidity at $0 \%$ with silica gel, as measured by a humidity meter. The temperature was controlled in the range $17-33^{\circ} \mathrm{C}$ by changing the temperature of the entire room. The chamber was repurged with $\mathrm{N}_{2}$ after the temperature was changed, to maintain a pressure of $1 \mathrm{~atm}$, so the experiment is at constant pressure in this range. Above $33^{\circ} \mathrm{C}$, the room was kept at $33^{\circ} \mathrm{C}$ and the temperature in the chamber was altered by the use of a small lamp within the chamber; below $17^{\circ} \mathrm{C}$, the chamber was chilled with ice [21].

The clamped end of the cantilever was driven with an amplitude $A$ (about $0.05 \mathrm{~nm}$ ) at the resonant frequency (about $6 \mathrm{kHz}$ ), while the deflection of cantilever $z$ the amplitude of deflection $R$, and the phase of the cantilever deflection relative to the drive $\theta$ were measured using a lock-in amplifier while the plate was driven toward the cantilever. The damping coefficient $D$ was obtained from $R$ and $\theta$ as a function of separation, assuming the cantilever executes simple harmonic motion, modeled as

$$
m \ddot{z}+D(s) \dot{z}+\left[k-F^{\prime}(s)\right] z=k A \cos (\omega t),
$$

where $k$ is the spring constant, $m$ is the mass, $\omega$ is the drive frequency, $F^{\prime}$ is the stiffness of any material in parallel with the spring, and $s$ is the separation between the sphere and plate. We measure the resonant frequency at infinite separation, $\omega_{0}(\infty)$, and then drive the cantilever at this frequency at all separations. For a cantilever driven at $\omega_{0}(\infty)$, the damping is given by [22]

$$
D(s)=-\frac{k}{\omega_{0}(\infty)} \frac{A}{R(s)} \sin \theta(s)
$$

The slip length was determined from $D(s)$ using [23],

$$
D_{\mathrm{lub}}(s)=\frac{6 \pi \eta r^{2}}{s} f^{*}(s, b),
$$

where $D_{\text {lub }}(s)=D(s)-D(\infty), \eta$ is dynamic viscosity of the nitrogen gas, $r$ is the radius of the sphere, and $b$ is the slip length (assumed identical on both surfaces). $f^{*}$ is an algebraic expression derived by Vinogradova [23] and written in the form used here in Ref. [18]. The dynamic viscosity is a function of temperature (varying by about $10 \%$ at $1 \mathrm{~atm}$ in the range $9-42{ }^{\circ} \mathrm{C}$ for $\mathrm{N}_{2}$ ) and was adjusted accordingly.

The key result of our work is that the accommodation coefficient is a function of the temperature for a single film, as shown in Fig. 1, and thus the accommodation coefficient and slip flow boundary condition can be altered by changing the temperature. Therefore, in situ modification of the flow boundary condition is possible, leading to control of flow for a constant geometry and gas type. Since $\lambda$ is an input in the calculation of $\sigma$, we accounted for the linear increase in $\lambda$ with temperature according to the kinetic theory of gases [24].

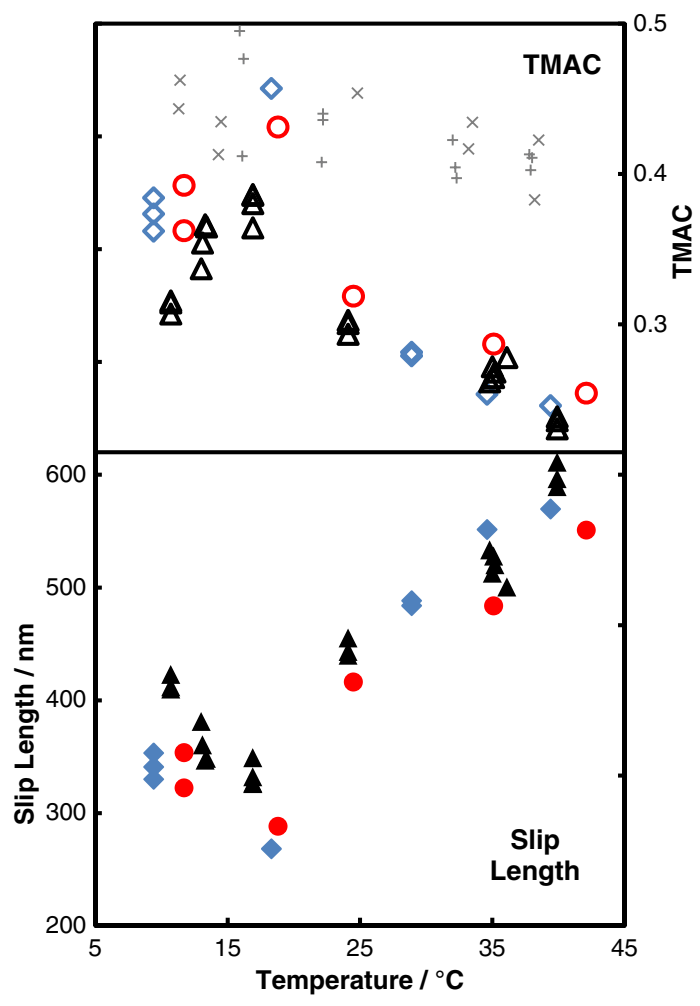

FIG. 1 (color online). Measured TMAC and slip length of 1 atm nitrogen gas on OTS-coated glass. Different symbols represent different sphere-plate pairs prepared by the same method (set 1: diamonds, set 2: circles, set 3: triangles). The slip length data in the bottom pane were used to calculate the accommodation coefficients for the same sphere-plate pair in the top pane. + and $\times$ symbols show the TMAC for two sets of bare glass surfaces. 
In detail, there is a maximum in TMAC of $\approx 0.44$ at $18{ }^{\circ} \mathrm{C}$. TMAC decreases to 0.25 at $42^{\circ} \mathrm{C}$, and $\approx 0.3$ at $9{ }^{\circ} \mathrm{C}$. There is a systematic relative uncertainty of about $3 \%$ in all fitted slip lengths that arises from error in the radius and spring constant. This propagates into an uncertainty of about 3\% in the TMAC. There is an additional relative uncertainty of $3 \%$ in each separate measurement of slip length, which propagates into $\sim 3 \%$ in the TMAC. For each sample, we repeated measurements at the same temperature (see Fig. 1) and the average variability in repeat measurements was about $\pm 11 \mathrm{~nm}$, or 0.01 in TMAC, which is much smaller than the overall temperature-induced decrease in TMAC of about 0.2. Note that the adjustments for the variation in viscosity and $\lambda$ with temperature are much smaller than the effects in Fig. 1. Changes in TMAC for bare glass surfaces (no film) over the same temperature range were very slight, as shown in the top part of Fig. 1.

Figure 1 shows results from three sphere-plate pairs. There is a significant variation from pair to pair, but all pairs show the same overall trend with a maximum in the TMAC at about $18^{\circ} \mathrm{C}$. This variation in TMAC is mainly attributed to slight variation in sample preparation. Note also that very similar results are obtained when measuring the TMAC at the same temperature twice, but with several different temperatures in between; i.e., the TMAC can be changed reversibly on the same film.

We now examine hypotheses to explain the measured variation in TMAC. The first hypothesis is that the OTS film becomes less rough with increased temperature, and that this causes a smaller fraction of gas molecules to experience a change in the tangential component of velocity after colliding with the solid. This loss of roughness may be due to loss of crystalline patches of OTS chains during melting. To test this hypothesis, we have measured the roughness of two OTS films as a function of temperature using AFM tapping mode (MFP-3D, Asylum Research; ORC-8 Cantilever A, Bruker), a peltier device to alter the temperature and a glove bag purged with dry air to control the humidity. Fig. 2 shows the measured root mean squared (rms) roughness of two OTS-coated glass plates, measured over an area of $(10 \mu \mathrm{m})^{2}$, which enables us to measure roughness on a lateral scale of about $40 \mathrm{~nm}$. We make two observations. First, the rms roughness is of the same order as a nitrogen molecule radius, $\sim 250 \mathrm{pm}$ [24], so the roughness has been changed on a scale that is likely to affect molecular collisions. Second, both plates show a monotonic decrease in rms roughness from about 800 to $50 \mathrm{pm}$ in the temperature range 16 to $41^{\circ} \mathrm{C}$, which is consistent with earlier observations of a more liquidlike conformation distribution at higher temperature. Thus the measured decrease in accommodation coefficient at temperatures above $18^{\circ} \mathrm{C}$ is correlated with a decrease in roughness.

The measured decrease in roughness does not explain the decrease in TMAC that occurs below $18^{\circ} \mathrm{C}$. Our hypothesis is that the film stiffness experienced by the nitrogen

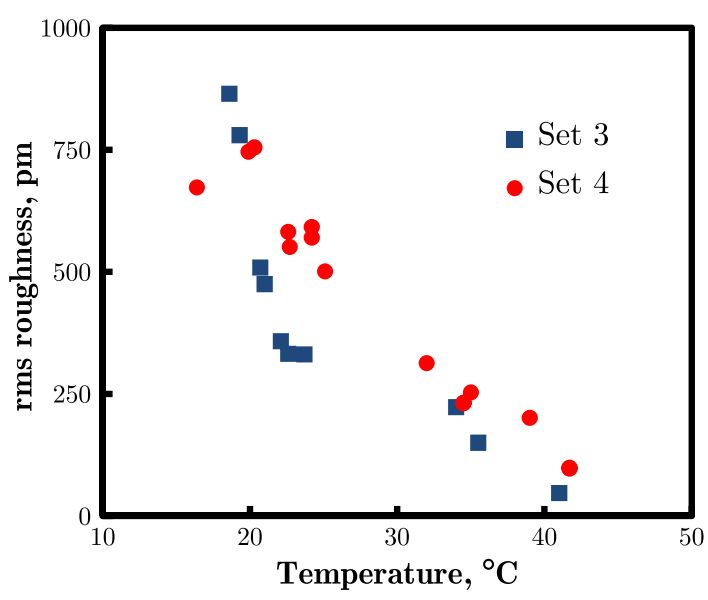

FIG. 2 (color online), rms roughness of two OTS-coated glass surfaces as a function of temperature.

molecule also decreases with increasing temperature when the chains melt. The decrease in film stiffness leads to an increase in the accommodation coefficient. We cannot examine the stiffness on the scale of a nitrogen molecule, but we can examine the overall stiffness on the scale of the sphere, which should depend on the local stiffness.

The stiffness of the film was determined by measuring the amplitude and phase of the cantilever driven at $\omega_{0}(\infty)$ after the sphere makes contact with the solid. In the simple harmonic oscillator model, any spring in parallel to the cantilever has a force gradient (stiffness) given by [22]

$$
F^{\prime}(s)=-\frac{k A}{R(s)} \cos \theta(s) .
$$

We are interested in the stiffness of the film, which we determined from $R$ and $\theta$ immediately after initial contact of the plate. This contact can be identified by a sudden jump in the deflection signal followed by a steep repulsion. Our interest lies in the region -0.5 to $0 \mathrm{~nm}$, where the film is gently compressed. The low load limit is of interest because the film is lightly loaded during the lubrication experiment. The data are noisy, but there is a trend to a stiffer film at lower temperature [Fig. 3(b)], and, in particular, an increase in stiffness between 19 and $12^{\circ} \mathrm{C}$. Thus, the small decrease in accommodation coefficient (increased conservation of gas momentum in the tangential direction) when the temperature drops from 19 and $12{ }^{\circ} \mathrm{C}$ correlates with an increase in stiffness of the film.

So far we have elucidated two trends for the OTS film as a function of temperature: the film becomes less rough and less stiff as temperature increases. We expect these two properties of the film have opposite effects on TMAC, which could lead to a maximum. The question now is why they should each control the TMAC in different regimes. Our simple idea is that increases in roughness only affect TMAC up to a certain magnitude of roughness. First, we point out that our AFM cannot be cooled, so only have roughness data at temperatures greater than $16-18^{\circ} \mathrm{C}$, and 

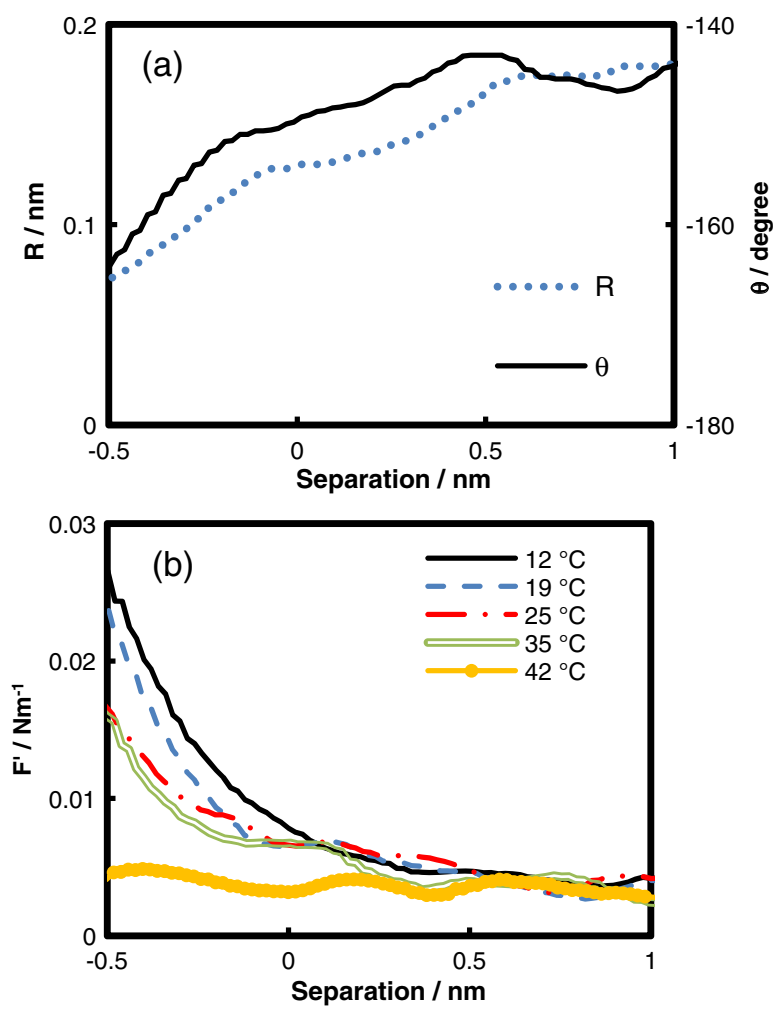

FIG. 3 (color online). Stiffness of OTS film, $F^{\prime}$, with probe in contact. (a) $R$ (left vertical axis), and $\theta$ (right vertical axis) used to calculate stiffness at $25^{\circ} \mathrm{C}$. The lock-in amplifier time constant of $10 \mathrm{~ms}$ leads to data averaging of the $R$ and $\theta$ over a distance of $0.2 \mathrm{~nm}$ in this figure. (b) Stiffness as a function of temperature, calculated using Eq. (5).

it is not clear that the film is increasing in roughness below $18^{\circ} \mathrm{C}$. Also, once the film is fully crystallized, there is no clear mechanism for further roughening. Even if the film were to become rougher, it is not clear that this would continue to affect TMAC indefinitely because we expect roughness on a scale much greater than the nitrogen molecule radius $(\sim 250 \mathrm{pm})$ to have a diminishing effect. From Fig. 2 we see that the film roughness has grown to three molecular radii at $18{ }^{\circ} \mathrm{C}$, and there may be no further effect of roughness at that point.

Our final question is on the magnitude of flow changes that can occur by changing the flow boundary condition. We calculated the volume rate of flow $\dot{V}$ through a cylindrical tube with the assumptions of the Hagen-Poiseuille equation, except that partial slip is allowed. This example illustrates flow through a pore in a membrane. Ignoring the pressure dependence of the slip length via the mean free path [Eq. (1)]:

$$
\dot{V}(b)=\frac{\pi\left(P_{0}-P_{L}\right) R_{T}^{4}}{8 \mu L}\left(1+\frac{4 b}{R_{T}}\right),
$$

where $P_{0}$ is the inlet pressure, $P_{L}$ is the outlet pressure, $R_{T}$ is the radius of the tube, and $L$ is the length of the tube. For a given pressure drop, length of tube, radius, and viscosity

$$
\frac{\dot{V}\left(b_{1}\right)}{\dot{V}\left(b_{2}\right)}=\frac{\left(R_{T}+4 b_{1}\right)}{\left(R_{T}+4 b_{2}\right)},
$$

where $b_{1}$ and $b_{2}$ represent the slip lengths on two different surfaces. For example, assuming $b_{1}$ has the same slip length as OTS at $40{ }^{\circ} \mathrm{C}(600 \mathrm{~nm})$ and $b_{2}$ has the same slip length as OTS at $18{ }^{\circ} \mathrm{C}(290 \mathrm{~nm})$, and a tube radius of $1000 \mathrm{~nm}$, the volumetric flow increases by about 50\%. If we account for the variation in $b$ is a function of pressure along the tube, and the TMAC is assumed independent of pressure, the flow increases by about $45 \%$. Thus the changes in slip length observed in this Letter will cause significant changes in flow through a microscopic cylinder.

In summary, we conclude from this work that the slip length at the solid-gas interface can be altered in situ when a responsive surface film is added to a glass solid. In this case, we cause the response for $\mathrm{N}_{2}$ at 1 atm by changing the temperature. After accounting for the small effect of temperature on the mean free path, we also show that the tangential momentum accommodation coefficient can be altered in situ by changing the temperature. We attribute the main changes in accommodation coefficient to changes in surface roughness that are produced in the film by temperature changes. The properties of surface films can also be changed by other stimuli, so changes in tangential accommodation and concomitant flow rates should also be affected by a variety of stimuli such as light or electric fields. The magnitudes of changes in flow are significant for channels with dimensions on the order of $1 \mu \mathrm{m}$ in size.

The importance of this work is that it demonstrates the ability to control gas flow even for fixed geometry and gas properties. It is the first demonstration of a physical principal that could be used for applications such as controlling the flight of miniature or very high altitude aircraft simply by altering the resistance on selected parts of the aircraft, or for throttling the flow through a microfluidic device without the use of any moving parts.

This research was funded by the National Science Foundation via Grant No. CBET-0959228. The authors also thank Dean Mastropietro for assistance with the AFM equipment. We thank Professor John Morris, Virginia Tech, for useful discussions.

*To whom all correspondence should be addressed. wducker@vt.edu

[1] Handbook of Chemistry and Physics, edited by W. M. Haynes (CRC Press, Boca Raton, 2013), 94th ed.

[2] R.W. Wang, J. Guo, G. Baran, and S. L. Wunder, Langmuir 16, 568 (2000).

[3] M. Ho and J. E. Pemberton, Anal. Chem. 70, 4915 (1998).

[4] R. G. Nuzzo, E. M. Korenic, and L. H. Dubois, J. Chem. Phys. 93, 767 (1990).

[5] G. Karniadakis, A. Beskok, and N. Aluru, Microflows and Nanoflows (Springer-Verlag, New York, 2005). 
[6] A. Frangi, G. Spinola, and B. Vigna, Int. J. Numer. Methods Eng. 68, 1031 (2006).

[7] C. D. F. Honig, J. E. Sader, P. Mulvaney, and W. A. Ducker, Phys. Rev. E 81, 056305 (2010).

[8] F. Ezquerra Larrodé, C. Housiadas, and Y. Drossinos, Int. J. Heat Mass Transfer 43, 2669 (2000).

[9] J. C. Maxwell, Philos. Trans. R. Soc. London 170, 231 (1879).

[10] C. R. Lilley and J.E. Sader, Proc. R. Soc. A 464, 2015 (2008).

[11] For nitrogen gas at $1 \mathrm{~atm}$ and the slip length in the range 300-1000 nm, the TMAC calculated using Maxwell's equation is systematically about $5 \%$ greater than when calculated using Lilley and Sader's equation.

[12] G. Binnig, C. F. Quate, and C. Gerber, Phys. Rev. Lett. 56, 930 (1986).

[13] W. A. Ducker, T. J. Senden, and R. M. Pashley, Nature (London) 353, 239 (1991).

[14] C. D. F. Honig, J. E. Sader, P. Mulvaney, and W. A. Ducker, Phys. Rev. E 81, 056305 (2010).

[15] J. Laurent, A. Drezet, H. Sellier, J. Chevrier, and S. Huant, Phys. Rev. Lett. 107, 164501 (2011).
[16] C. Lissandrello, V. Yakhot, and K. L. Ekinci, Phys. Rev. Lett. 108, 084501 (2012).

[17] C. D. F. Honig and W. A. Ducker, J. Phys. Chem. C 114, 20114 (2010).

[18] D. Seo, D. Mastropietro, and W. A. Ducker, J. Phys. Chem. C 117, 6235 (2013).

[19] E. B. Arkilic, K. S. Breuer, and M. A. Schmidt, J. Fluid Mech. 437, 29 (2001).

[20] J. Maurer, P. Tabeling, P. Joseph, and H. Willaime, Phys. Fluids 15, 2613 (2003).

[21] Between $33^{\circ} \mathrm{C}$ and $41^{\circ} \mathrm{C}$, and between $17^{\circ} \mathrm{C}$ and $11^{\circ} \mathrm{C}$ the pressure was not equilibrated with the atmosphere, so the pressure will be about $2.5 \%$ greater at $41{ }^{\circ} \mathrm{C}$ and $2.7 \%$ lower than $1 \mathrm{~atm}$ due to the increase or decrease in termperature. This change in pressure due to heating or cooling at constant volume does not change the mean free path.

[22] W. A. Ducker and R. F. Cook, Appl. Phys. Lett. 56, 2408 (1990).

[23] O. I. Vinogradova, Langmuir 11, 2213 (1995).

[24] P.W. Atkins, Physical Chemistry (Oxford University Press, Oxford, 1994), estimated from the van der Waals constant in Table 1.5. 